Clinical Research

\title{
COVID-19 and fat embolism: a hypothesis to explain the severe clinical outcome in people with obesity
}

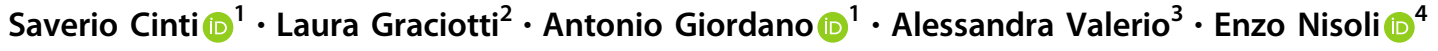

Received: 23 April 2020 / Revised: 25 May 2020 / Accepted: 29 May 2020 / Published online: 8 June 2020

(c) Springer Nature Limited 2020

As the number of confirmed cases of COVID-19 surges past 4.7 million globally and deaths surpass 315,000 , clinicians and pathologists are untiringly working to comprehend the damage produced by the coronavirus through the body. They are understanding that, yet the lungs are massively deranged, COVID-19 infection can outspread to many organs including the blood vessels and heart, gut, brain, and kidneys [1]. A systemic therapeutic approach is necessary; we thus analyzed a global interpretation of multi-organ symptoms and signs to propose a new pathogenetic hypothesis, with potential drug therapy implications, in COVID-19 people with obesity. Recent studies point to obesity as a critical risk factor for being hospitalized with COVID-19 [2-4]. Indeed, a high prevalence of obesity has been observed in COVID-19 patients requiring invasive mechanical ventilation [5], a robust proxy of SARS-CoV2 severity. In patients under the age of 60 , those with obesity were at almost double risk of being admitted to critical care when compared with normal-weight patients [6].

Of note, coagulation and fibrinolysis are altered in obesity, mainly due to a high plasma concentration of multiple prothrombotic factors-including plasminogen activator inhibitor-

Saverio Cinti

s.cinti@staff.univpm.it

$\triangle$ Enzo Nisoli

enzo.nisoli@unimi.it

1 Department of Experimental and Clinical Medicine, Center of Obesity, Marche Polytechnic University, 60020 Ancona, Italy

2 Department of Clinical and Molecular Sciences, Section of Experimental Pathology, Marche Polytechnic University, 60020 Ancona, Italy

3 Department of Molecular and Translational Medicine, University of Brescia, 25123 Brescia, Italy

4 Department of Biomedical Technology and Translational Medicine, Center for the Study and Research on Obesity, University of Milan, 20129 Milan, Italy
1, fibrinogen, von Willebrand factor, and factor VII-than nonobese subjects [7]. Although the cause of pro-thrombotic milieu in obese organs is poorly understood, the increased plasma levels of interleukin-6 (IL-6), tumor necrosis factor- $\alpha$ (TNF- $\alpha$ ), and leptin seem to play important roles [7]. Obesity is associated with increased expression of inflammatory mediators in adipose tissue and this inflammation interferes with glucose metabolism (also named metaflammation) in both animals and humans, mainly due to macrophage infiltration $[8,9]$. This macrophage infiltration originates from adipocyte necrotic death, a consequence of the obesity-induced adipocyte hypertrophy and inflammasome activation [10]; importantly, the white adipocytes of visceral depots have a lower critical death size, with a consequent higher inflammatory response, than subcutaneous fat cells [11]. Thus, abundant free lipid droplets-released from dead adipocytes-are present in the extracellular space of adipose tissue (or adipose interstitium), particularly in visceral fat, to be removed by macrophages [10], whose chronic activation causes the release of abnormal amounts of IL- 6 and TNF- $\alpha$. Also, adipocyte size is a determinant of leptin production, and hyperleptinemia has been related to increased thrombotic risk in obesity [12]. Accordingly, the adipocyte hypertrophy and consequent adipose metaflammation are responsible for the altered coagulation observed in people with obesity [13].

SARS-CoV-2 (Fig. 1) contaminates multiple cell types in different organs, and-since adipocytes express the membrane-bound glycoprotein angiotensin-converting enzyme 2 (ACE2) [14], the critical receptor mediating the virus entry-there is no reason to think that the adipose organ is saved. SARS-CoV-2 fat infiltration could worsen adipose inflammatory status in the expanded adipose tissues of obese patients, as ACE2 is more expressed in obese mice [14]. In addition, the SARS-CoV-2-infected adipocytes would massively undergo necrotic death, as shown in other cell types [15], exacerbating lipid remnant accumulation in the adipose interstitium, with free lipid droplets that, in turn, could predispose COVID-19 patients with obesity to fat embolism syndrome (FES). FES is a miscellanea of 


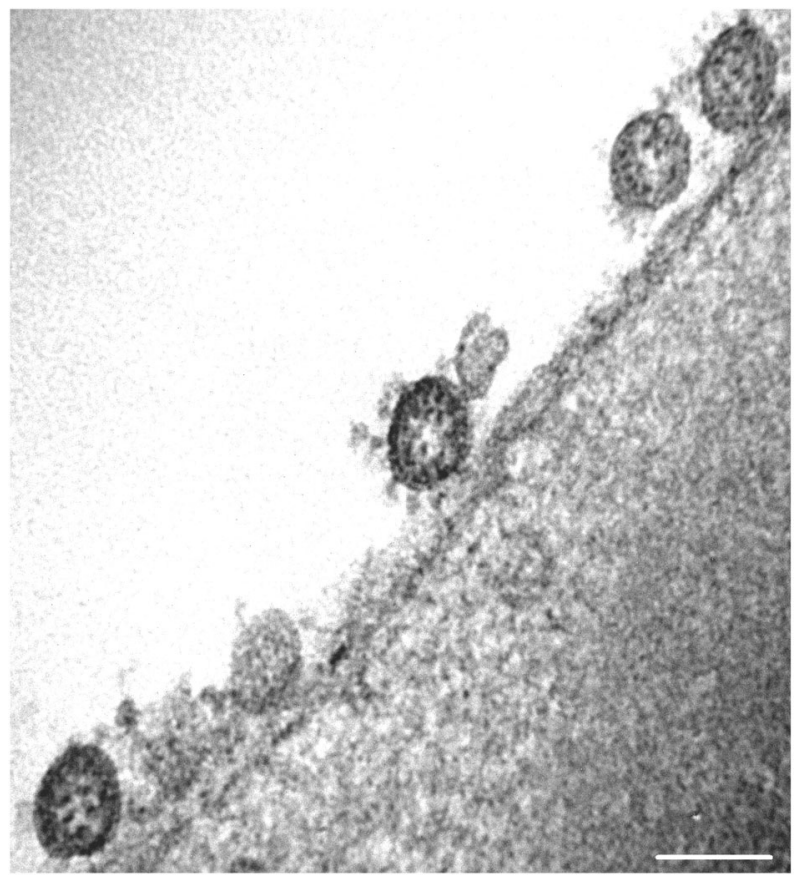

Fig. 1 Electron microscopy of isolated SARS-CoV-2 at the external plasma membrane of Vero E6 cell in culture. Bar: $100 \mathrm{~nm}$ (in collaboration with Prof. Patrizia Bagnarelli and Prof. Stefano Menzo, Department of Biomedical Sciences and Public Health, Virology, Marche Polytechnic University_United Hospitals of Ancona, Italy).

respiratory, hematological, neurological, and cutaneous symptoms and signs - related to trauma or multiple surgical and medical complications - and due to fat embolism in the microcirculation [16]. Of note, obesity per se was never described as a cause of FES [17-19]. Clinical diagnosis of FES is difficult, especially when unsuspected in the absence of severe trauma or bone fractures. Noteworthy, the diffuse ground-glass opacities described in COVID-19 lung computed tomography scans are strongly reminiscent of the bilateral pulmonary patchy infiltrates leading FES patients to develop acute respiratory distress syndrome [20]. Further, primary FES diagnostic criteria include petechial rash and central nervous system signs, that have recently been observed in COVID-19 case [21, 22]. Strikingly, we have found clear evidence of fat embolism in autoptic lung specimens from two adult COVID-19 patients (one male and one female) with overweight (Fig. 2), suggesting that this might be a very relevant pathological event, with severe consequences for drug management. Of note, lipid-like structures were also found in the lumen of mesenteric venous vessels of one of the two patients (not shown).

These findings require further investigation, actively forwarded in diverse directions to confirm these our preliminary observations, including to search for fat embolism in other organs besides lung in COVID-19 patients. However, we suggest that in SARS-CoV-2-related pneumoniaparticularly in people with overweight or obesity-

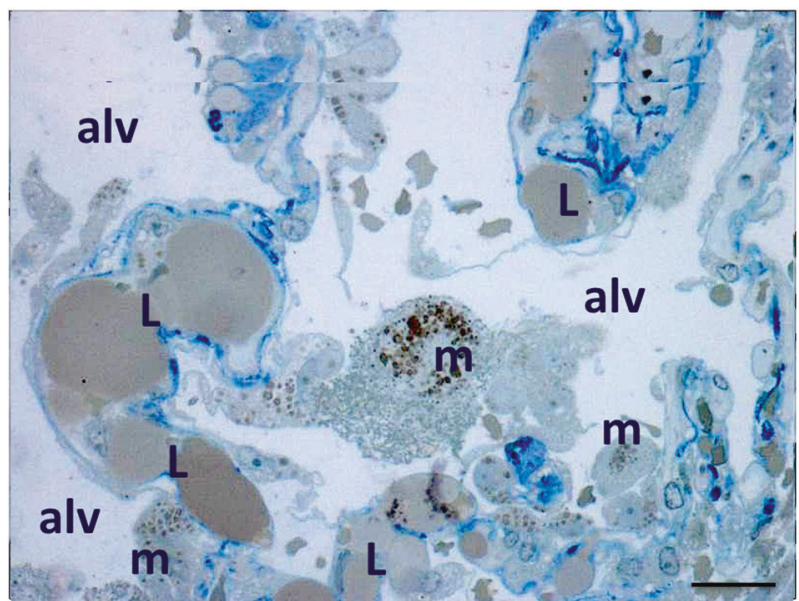

Fig. 2 Light microscopy of lung biopsy from an adult overweight patient died by COVID-19. Resin-embedded and toluidine bluestained section (procedure inducing a characteristic yellow staining of lipids). Note the numerous large lipid droplets (L) into the capillary lumen of alveolar septa. Alveolar macrophages are filled with cytoplasmic lipid droplets. Alv: aveolar space, m: macrophages. Bar: 15 microns (in collaboration with Prof. Adriano Tagliabracci and Dr Mauro Pesaresi, Department of Biomedical Sciences and Public Health, Legal medicine, Marche Polytechnic University-United Hospitals of Ancona, Italy).

clinicians should focus their awareness and cautions to the therapeutic interventions tried, though with varying success, in FES patients, such as controlled humidified oxygen therapy, low molecular weight heparin, corticosteroids, and other anti-inflammatory drugs $[16,20]$. Heparin, in particular, known to stimulate lipase activity-thus favouring the clearance of circulating lipids-has been proposed for FES treatment with contradictory results $[16,20]$. Indeed, the beneficial effects of lipase activation might be counterbalanced by the resultant increase in free fatty acids (FFAs) that can exacerbate FES-related inflammation [16]. If increased levels of FFAs are a crucial part of FES pathogenesis, then activation of lipase is a potentially dangerous therapeutic intervention. Besides, the risk of bleeding cannot be ignored in COVID-19 patients, mainly those with overweight or obesity, even treated with low-dose heparin. Aspirin, in turn, has been suggested as an FES prophylactic agent, given its ability to prevent gas exchange abnormalities [23] beyond blocking the production of proinflammatory and pro-thrombotic thromboxane-which occurs in animal models of FES [16] - yet its use has not been considered in most recent studies. Further, though evidence in favor of their routine administration is lacking, corticosteroids have been considered in cases of fulminant FES [20], and this can support their use in COVID-19 patients only in selected cases of compelling indications [24], with predictable limitations in people with overweight or obesity. Finally, cardiopulmonary resuscitation 
procedures should be afforded with caution in suspected FES linked to SARS-CoV-2 infection, since they could predispose to fat embolization [25] adding further questions to a critical, yet unsolved issue in the COVID-19 patients with overweight or obesity [26]. Thus, our pathogenetic hypothesis, which considers fat necrosis and metaflammation as likely crucial contributors to SARS-CoV-2-induced FES, highlights further critical points in seeking proper drugs within the COVID-19 therapeutic arsenal, particularly in patients with overweight or obesity. Studies exploring the pathophysiological consequences of SARS-CoV2 infection of adipose tissue, the diagnostic investigation of fat embolism, and the clinical relevance of visceral obesity in the COVID-19 scenario are urgently needed.

Acknowledgements SC is supported by Progetti di Rilevante Interesse Nazionale (PRIN, 2018). EN and AV are supported by a grant from the Cariplo Foundation (2016-1006). EN is supported by a Professional Dietetics award (Milan, Italy).

\section{Compliance with ethical standards}

Conflict of interest The authors declare that they have no conflict of interest.

Publisher's note Springer Nature remains neutral with regard to jurisdictional claims in published maps and institutional affiliations.

\section{References}

1. Wadman M, Couzin-Frankel J, Kaiser J, Matacic C. How does coronavirus kill? Clinicians trace a ferocious rampage through the body, from brain to toes. Science. 2020. https://doi.org/10.1126/ science.abc3208.

2. Dietz W, Santos-Burgoa C. Obesity and its implications for COVID-19 mortality. Obesity. 2020. https://doi.org/10.1002/oby. 22818.

3. Finer N, Garnett SP, Bruun JM. COVID-19 and obesity. Clin Obes. 2020. https://doi.org/10.1111/cob.12365.

4. Kassir R. Risk of COVID-19 for patients with obesity. Obes Rev. 2020;21:e13034. https://doi.org/10.1111/obr.13034.

5. Simonnet A, Chetboun M, Poissy J, Raverdy V, Noulette J, Duhamel A, et al. High prevalence of obesity in severe acute respiratory syndrome coronavirus-2 (SARS-CoV-2) requiring invasive mechanical ventilation. Obesity. 2020. https://doi.org/10. 1002/oby.22831.

6. Lighter J, Phillips M, Hochman S, Sterling S, Johnson D, Francois $\mathrm{F}$, et al. Obesity in patients younger than 60 years is a risk factor for Covid-19 hospital admission. Clin Infect Dis. 2020;ciaa415. https://doi.org/10.1093/cid/ciaa415.

7. Blokhin IO, Lentz SR. Mechanisms of thrombosis in obesity. Curr Opin Hematol. 2013;20:437-44. https://doi.org/10.1097/MOH. 0b013e3283634443.

8. Gregor MF, Hotamisligil GS. Inflammatory mechanisms in obesity. Annu Rev Immunol. 2011;29:415-45. https://doi.org/10. 1146/annurev-immunol-031210-101322.
9. Saltiel AR, Olefsky JM. Inflammatory mechanisms linking obesity and metabolic disease. J Clin Invest. 2017;127:1-4. https:// doi.org/10.1172/JCI92035.

10. Cinti S, Mitchell G, Barbatelli G, Murano I, Ceresi E, Faloia E, et al. Adipocyte death defines macrophage localization and function in adipose tissue of obese mice and humans. J Lipid Res. 2005;46:2347-55. https://doi.org/10.1194/jlr.M500294-JLR200.

11. Murano I, Barbatelli G, Parisani V, Latini C, Muzzonigro G, Castellucci $\mathrm{M}$, et al. Dead adipocytes, detected as crown-like structures, are prevalent in visceral fat depots of genetically obese mice. J Lipid Res. 2008;49:1562-8. https://doi.org/10.1194/jlr. M800019-JLR200.

12. Konstantinides S, Schäfer K, Koschnick S, Loskutoff DJ. Leptindependent platelet aggregation and arterial thrombosis suggests a mechanism for atherothrombotic disease in obesity. J Clin Invest. 2001;108:1533-40. https://doi.org/10.1172/JCI13143.

13. Hotamisligil GS. Inflammation, metaflammation and immunometabolic disorders. Nature. 2017;542:177-85. https://doi.org/10. 1038/nature21363.

14. Gupte M, Boustany-Kari CM, Bharadwaj K, Police S, Thatcher S, Gong MC, et al. ACE2 is expressed in mouse adipocytes and regulated by a high-fat diet. Am J Physiol Regul Integr Comp Physiol. 2008;295:R781-8. https://doi.org/10.1152/ajpregu.00183.2008.

15. Tan YJ, Lim SG, Hong W. Regulation of cell death during infection by the severe acute respiratory syndrome coronavirus and other coronaviruses. Cell Microbiol. 2007;9:2552-61.

16. Mellor A, Soni N. Fat embolism. Anaesthesia. 2001;56:145-54. https://doi.org/10.1046/j.1365-2044.2001.01724.x.

17. Rothberg DL, Makarewich CA. Fat embolism and fat embolism syndrome. J Am Acad Orthop Surg. 2019;27:e346-55. https://doi. org/10.5435/JAAOS-D-17-00571.

18. Meng Y, Zhang M, Ling H, Huang S, Miao Q, Yu Y, et al. Nontraumatic multiple-organ fat embolism: an autopsy case and review of literature. Am J Forensic Med Pathol. 2020;41:131-4. https://doi.org/10.1097/PAF.0000000000000544.

19. Fukumoto LE, Fukumoto KD. Fat embolism syndrome. Nurs Clin North Am. 2018;53:335-47. https://doi.org/10.1016/j.cnur.2018. 04.003.

20. Kosova E, Bergmark B, Piazza G. Fat embolism syndrome. Circulation. 2015;131:317-20. https://doi.org/10.1161/ CIRCULATIONAHA.114.010835

21. Recalcati S. Cutaneous manifestations in COVID-19: a first perspective. J Eur Acad Dermatology Venereol. 2020. https://doi.org/ 10.1111/jdv.16387.

22. Mao L, Jin H, Wang M, Hu Y, Chen S, He Q, et al. Neurologic manifestations of hospitalized patients with coronavirus disease 2019 in Wuhan, China. JAMA Neurol. 2020. https://doi.org/10. 1001/jamaneurol.2020.1127.

23. Shier MR, Wilson RF, James RE, Riddle J, Mammen EF, Pedersen HE. Fat embolism prophylaxis: a study of four treatment modalities. J Trauma. 1977;17:621-9.

24. Sanders JM, Monogue ML, Jodlowski TZ, Cutrell JB. Pharmacologic treatments for coronavirus disease 2019 (COVID-19): a review. JAMA. 2020. https://doi.org/10.1001/jama.2020.6019.

25. Turkmen Samdanci E, Celik MR, Pehlivan S, Celbis O, Turkkan D, Ozdemir Kara D, et al. Histopathological evaluation of autopsy cases with isolated pulmonary fat embolism (IPFE): is cardiopulmonary resuscitation a main cause of death in IPFE? Open Access Emerg Med. 2019;1:121-7. https://doi.org/10.2147/ OAEM.S194340.

26. Fritz Z, Perkins GD. Cardiopulmonary resuscitation after hospital admission with covid-19. BMJ. 2020;369:m1387. https://doi.org/ 10.1136/bmj.m1387. 